Prof. Dr. med. Thomas Lehrnbecher, Frankfurt

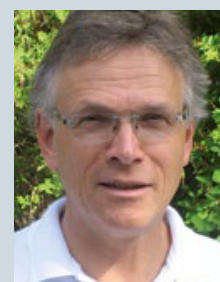

Dr. med. Ulrich Mutschler, Hildesheim

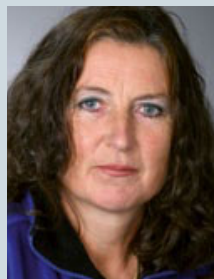

Dr. med. Kirsten Stollhoff, Hamburg

\section{Autismus und ADHS häufig gepaart}

Über viele Jahre wurde eine Assoziation übersehen, ja sogar formell gemäß Definition durch das internationale Klassifikationssystem ausgeschlossen: Autismus und ADHS. In einer Übersicht werden aktuelle Daten vorgestellt.
$\mathrm{N}$ euere Studien weisen daraufhin, dass eine Überlappung von Autismus und ADHS in ca. $50 \%$ der betroffenen Kinder vorliegt. Genetische Untersuchungen legen ein polygenetisches Geschehen nahe. Assoziationsstudien finden 16 ,single nucleotid“-Polymorphismen, die für beide Störungen relevant sein können. Und schließlich wurden in neurobiologischen Untersuchungen zwar deutliche Unterschiede beobachtet, bei beiden Störungen ist jedoch das Frontalhirn involviert.

Banaschewski T et al. Autismus und ADHS über die Lebensspanne. Nervenarzt 2011; 82: $573-81$

Kommentar: Klinisch fällt bei vielen Kindern mit einer Autismus-Spektrumstörung auf, dass sie auch an einer ADHS-Symptomatik leiden, umgekehrt werden bei einem Teil der Kinder mit ADHS autistische Verhaltensstörungen beobachtet. Die Autoren weisen daher zu Recht darauf hin, wie wichtig es ist, bei jedem Kind mit der Diagnose Autismus nach dem Vorliegen einer ADHS-Symptomatik zu suchen. Umgekehrt sollte bei Kindern mit der Diagnose ADHS auch das mögliche Vorliegen von autistischen Zügen abgeklärt werden.

Dies hat therapeutische Konsequenzen: Wie bei der idiopathischen ADHS, so ist auch bei der ADHS assoziiert mit einer autistischer Störung Methylphenidat wirksam. Jedoch sollte die Dosis bei Kindern mit Autismus und
ADHS sehr niedrig begonnen und in kleinen Schritten bis zum Wirkungseintritt gesteigert werden. In der Regel sind geringere Dosen erforderlich. Die Wirksamkeit ist geringer als bei Kindern mit reinem ADHS. Kinder mit ADHS und autistischen Zügen profitieren von dem bei Autismus eingesetzten Risperdal. Eine Kombination von Risperdal und Methylphenidat wird von den Autoren empfohlen.

Viele Mütter leiden darunter, dass ihr Kind mit ADHS kaum Freunde hat und wenig Interesse daran zeigt, sich zu verabreden. Sollten in solch einem Fall zusätzlich autistische Störungen diagnostiziert werden, so könnte dies Einfluss auf die Beratung hinsichtlich der Freizeitgestaltung des Kindes haben.

Dr. Kirsten Stollhoff

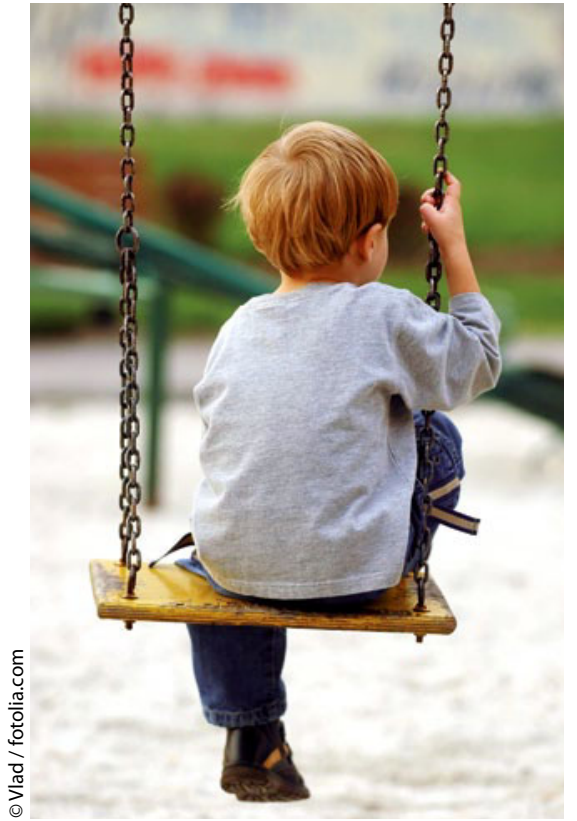

Kinder mit ADHS und autistischen Zügen werden anders therapiert als Kinder, die nur unter einer der Störungen leiden.

\section{Arzneischrank wird zur Falle}

Eine US-amerikanische Studie hat das Problem der Medikamentenvergiftung von Kindern untersucht. Ein Team von Notfallmedizinern hatte die Daten von rund 453.000 Kindern bis zu einem Alter von fünf Jahren ausgewertet, die zwischen 2001 und 2008 wegen einer befürchteten Medikamentenvergiftung in einer Notfallambulanz hatten behandelt werden müssen. 430.000 von ihnen hatten sich selbst vergiftet.

Knapp 250.000 Selbstvergiftungen gingen auf verschreibungspflichtige Präparate zurück. An erster Stelle standen Herz-Kreislauf-Medikamente, gefolgt von Analgetika überwiegend Opioide - und Sedativa bzw. Hypnotika. Bei den OTC-Medikamenten standen Analgetika an erster Stelle, v.a. Paracetamol, es folgten Erkältungsarzneien und Antihistaminika. Insgesamt stiegen die Zahlen für die vergiftungsbedingten Notrufe und Besuche einer Notaufnahme von 2001 bis 2008 erheblich an. Dr. Robert Bublak

Bond GR et al. J Pediatr 2011; Sept 13 [Epub ahead of print] 